\title{
Regulation of the Fasting Enterohepatic Circulation of Bile Acids by the Migrating Myoelectric Complex in Dogs
}

\author{
R. B. Scott, S. M. Strasberg, T. Y. El-Sharkawy, and N. E. Diamant, \\ Departments of Medicine, Surgery, Physiology, and Institute of Medical \\ Science, University of Toronto, Toronto, Ontario, Canada
}

A BSTRACT The purpose of this study was to correlate the fasting enterohepatic circulation (EHC) of bile acids with the migrating myoelectric complex. Four dogs were surgically provided with a functional cholecystectomy, a duodenal cannula for direct vision cannulation of the common bile duct, and 12 bipolar electrodes implanted from stomach to terminal ileum. Bile was collected in equal-volume, timed aliquots over 6 to $10 \mathrm{~h}$. Aliquots were sampled and either returned to the duodenum for study of the intact EHC, or collected and retained in order to study the time course of the bile acid pool washout. In the washout experiments boluses of radiolabeled taurocholic acid were instilled into the duodenum before and after duodenal phase III of the migrating motor or myoelectric complex (MMC). In another group of experiments the bile acid pool was washed out and during a continuous duodenal infusion of taurocholic acid bile was collected to study the pattern of hepatic secretion. Results: (a) In all experiments, a single broad peak of bile flow and bile acid secretion occurred at 35-55\% of the MMC migration time. At this time the MMC had migrated to a point $70-85 \%$ of the distance along the small intestine. (b) During bile acid pool washout the peak of bile flow and bile acid secretion occurred with the distal migration of the first MMC and then bile flow and bile acid secretion rates decreased to a minimum and stabilized. (c) In bile acid pool washout experiments the radiolabeled bile acids instilled into the duodenum prior to duodenal phase III were secreted and peaked with peak endogenous bile acid secretion. The secretion of radiolabeled bile acids instilled into the duodenum after duodenal phase III was delayed until the subsequent cycle of the MMC. $88 \%$ of the bile acid pool collected over $6 \mathrm{~h}$ was secreted during the distal migration of the first MMC

Address reprint requests to Dr. Scott.

Received for publication 28 April 1982 and in revised form 25 October 1982.
$(2.4 \pm 0.4 \mathrm{~h})$. (d) After bile acid pool washout and during continuous duodenal infusion of taurocholic acid, hepatic bile flow and bile acid secretion continued to fluctuate with the same pattern observed with the EHC intact. Conclusions: (a) In the fasting state, the transport of intestinal bile acids to the liver is pulsatile rather than continuous and is determined by the MMC. Maximum hepatic secretion occurs when phase III of the MMC propels the intraluminal bile acid pool to its site of absorption in the distal small bowel. (b) The "housekeeping" action of the MMC is very efficient and clears $88 \%$ of the $6-\mathrm{h}$ washout bile acid pool in one pass.

\section{INTRODUCTION}

The enterohepatic circulation (EHC) ${ }^{1}$ of bile acids is dependent on intestinal motor activity for the transport of bile acids from the site of their release into the proximal duodenum to the site of their active absorption in the terminal ileum (1). Variation in the rate of hepatic bile acid secretion, and in the rate of delivery of bile acids to the duodenum are related to the condition of fasting or feeding (2-7). In the fed state the gallbladder contracts (8) and a continuous pattern of intestinal motor activity is induced (9). This results in a continuous circulation of the entire bile acid pool, high rates of hepatic bile acid secretion, and maximal rates of delivery of bile acids into the duodenum. During fasting, basal rates of hepatic bile acid secretion are present, and it has been shown that there is a continuing enterohepatic circulation of that portion of the bile acid pool not sequestered by the gallbladder (1013). Intestinal motor activity in the fasted state is not continuous, but is characterized by a cyclic pattern of motor activity termed the "migrating myoelectric complex," or MMC. An MMC is a recurring band of

\footnotetext{
${ }^{1}$ Abbreviations used in this paper: EHC, enterohepatic circulation; MMC, migrating motor or myoelectric complex; TLC, thin-layer chromatography.
} 
excitatory myoelectric (14), and associated contractile activity (15), progressing distally along the gut from lower esophageal sphincter to terminal ileum $(14,16$, 17). The MMC is responsible for the efficient but largely intermittant propulsion of intraluminal contents (18-22), and is cyclically correlated with pulsatile delivery of bile acids to the duodenum (23-26), and with gallbladder contraction (27). The MMC has been identified in all species of mammals investigated (14, $19,20,28-30)$, including man $(9,31)$.

The purpose of this study was to determine if fasting rhythmic fluctuations in hepatic bile flow and bile acid secretion occur as a result of the cyclical transport of intestinal bile acids by the MMC to terminal ileum. If this is so, maximum hepatic secretion should occur when the MMC propels the intraluminal bile acid pool to its site of active absorption in the terminal ileum. Hepatic bile acid secretion could not be studied in relation to myoelectric activity along the entire length of small bowel in man, and we therefore utilized a canine animal model. Portions of this work have previously been published in abstract form $(32,33)$.

\section{METHODS}

\section{Animal preparation}

Four healthy female mongrel dogs weighing $15-22 \mathrm{~kg}$ were used in this study. The experimental protocol was approved by the Toronto Western Hospital Animal Care Committee and conformed with the "Guiding Principles in the Care and Use of Animals" proposed in the Declaration of Helsinki.

Dogs were dewormed and preconditioned for 1 wk before surgery. Each animal was premedicated with atropine 0.6 $\mathrm{ml}$ i.v. Anesthesia was induced with sodium thiopental 10 $\mathrm{mg} / \mathrm{kg}$ i.v., and then maintained with nitrous oxide and halothane. Using sterile technique, a midline abdominal incision was made and the following three surgical procedures performed: (a) A functional cholecystectomy; $(b)$ insertion of a modified Thomas duodenal cannula to allow visualization of the opening of the common bile duct; $(c)$ implantation of electrodes for monitoring intestinal myoelectric activity.

The gallbladder, cystic duct, cystic artery, and common bile duct were identified and isolated. The cystic duct was ligated in two places with chromic cat gut sutures, and then divided between the sutures. All bile was then aspirated from the gallbladder.

Insertion of the duodenal cannula utilized standard experimental surgical techniques (34). Between experiments the cannula was closed with a screw-on cap.

The total length of the small intestine was measured and recorded. A teflon cannula bearing 12 pairs of electrodes was brought out through a stab incision made in the right lower quadrant of the abdominal wall and secured in place. Pairs of electrodes were implanted into the subserosal musculature at measured distances along the antimesenteric border of the small bowel, beginning at a site $1 \mathrm{~cm}$ beyond the pylorus and extending to a site $10 \mathrm{~cm}$ proximal to the ileocecal valve. The electrodes of each pair were placed $0.5 \mathrm{~cm}$ apart for bipolar recording. Before closure the small bowel length and electrode position were checked a second time.
Animals were given maintenance total parenteral nutrition for the first $5 \mathrm{~d}$ postoperatively, and then gradually advanced to standard solid laboratory chow and water ad lib. All dogs were allowed at least 2 postoperative wk to recover prior to recording intestinal myoelectric activity.

\section{Experimental design}

After the postoperative recovery period, animals were studied conscious, unrestrained, and after an 18 -h fast. Studies were performed a minimum of $5 \mathrm{~d}$ apart to allow equilibration of the bile acid pool between experiments. Fasting intestinal myoelectric activity was recorded throughout each experiment. There were three separate experimental protocols.

Protocol 1. Two 10-h experiments were performed in each of two dogs. In each experiment the common bile duct was cannulated and bile flow was returned to the duodenum until at least one MMC had completed a migration from duodenum to terminal ileum. Then equal-volume, timed aliquots were collected, sampled for bile acid assay, and returned to the duodenum; thereby maintaining an intact EHC. The small $(50 \mu \mathrm{l})$ samples taken from each aliquot for bile acid assay represented $<5 \%$ of bile flow, and thus did not constitute a significant interruption of the EHC (35).

Protocol 2. A total of 12 separate 8-10-h experiments were performed on three dogs. In each experiment the common bile duct was cannulated and bile flow returned to the duodenum while one control migration of an MMC was recorded. Then, equal-volume, timed aliquots of bile were collected, sampled for bile acid assay, and retained; thus interrupting the EHC. In eight experiments, $2-\mu \mathrm{Ci}$ radiolabeled $\left[{ }^{14} \mathrm{C}\right]$ sodium taurocholate was instilled into the duodenum prior to a period of intense duodenal electric spike activity (phase III of the MMC), and in seven experiments, $2 \mu \mathrm{Ci}$ radiolabeled $\left[{ }^{3} \mathrm{H}\right]$ sodium taurocholate was instilled into the duodenum just after termination of a period of intense duodenal electric spike activity (phase III of an MMC). In all cases, the time of instillation of the isotope coincided with the time at which bile collection began.

Protocol 3. Two 10-h experiments were performed in each of two dogs, after drainage of the bile acid pool. During each experiment the animals received a continuous duodenal infusion of sodium taurocholate in a solution of $5 \mathrm{~g} / \mathrm{liter}$ dextrose in water. The infusion was regulated at $0.5 \mathrm{ml} / \mathrm{min}$ by a microvolumetric infusion pump (IMED Canada Inc. model 965 ) and contained $40 \mu \mathrm{mol} / \mathrm{ml}$ of sodium taurocholate. Sodium taurocholate $(96 \%$ pure by thin-layer chromatography, TLC) was obtained from Calbiochem-Behring Corp., La Jolla, CA.

\section{Recording of intestinal myoelectric activity}

A cable connected the bipolar electrodes to bioelectric amplifiers (Hewlett Packard Co., Palo Alto, CA, model 8811A) with lower and upper cutoff frequencies of 0.05 and $300 \mathrm{~Hz}$, respectively. The electrical signals were simultaneously recorded on an eight-channel direct writing pen chart recorder (Hewlett-Packard Co., model 7858A) and on an eight-channel FM instrumentation tape recorder (Hewlett-Packard Co., model 8868A) for later replay and analysis.

To analyze fasting intestinal myoelectric activity, the taped records were played back at eight times the recording speed, high pass filtered to preferentially amplify spike potentials and recorded on a pen chart recorder running at $0.25 \mathrm{~mm} / \mathrm{min}$. This yielded a time-condensed recording 
from which the features of the migrating myoelectric complex were identified by visual inspection. Fig. 1 illustrates the typical condensed tracings of fasting electrical activity recorded from eight electrodes in one dog. Electrode locations are expressed as distance from the pylorus. The three MMC shown are recognized as cyclically recurring periods of spiking activity that migrate from duodenum to terminal ileum. The period of intense spiking activity (phase III) is identified as a dense segment of higher amplitude. Phase III is preceded by a period of irregular spiking activity (phase II), and followed by a phase of absent spike activity (phase I). The end of phase III is easily identified, and was chosen as a reference point. The time between the end of duodenal phase III activity of successive cycles was defined as the cycle period. The time required for progression of the MMC from the pylorus to ileocecal junction was defined as the migration time.

\section{Determination of bile flow and bile acid secretion rate}

The screw cap on the duodenal cannula was removed to visualize the opening of the common bile duct into the duodenum. The duct was cannulated with a 5-French polyvinyl chloride catheter. A rubber cork with side slot was then used to close the lumen of the duodenal cannula and fix the catheter in place. The free end of the catheter drained by gravity into a graduated pipette, accurate to $0.1 \mathrm{ml}$.

The experiments were intended to relate the hepatic bile acid secretion rate to the aboral migration of the MMC. For bile collections to accurately represent hepatic bile secretory rates, a correction for transit time down the biliary tree and collection tubing was necessary. The estimated "dead space" volume of the biliary tree and collection tubing was determined by the method of Haaki and Paumgartner (36), which was later validated in larger animals (dogs) by Barnhart and Combes (37). The technique depends upon the biliary clearance of an intravenous bolus of radiolabeled sodium taurocholate. A dead space correction technique described previously (38) was then used. To reduce sampling errors due to dead space, sample volumes were twice the estimated dead space. Bile flow was calculated as the fixed volume of

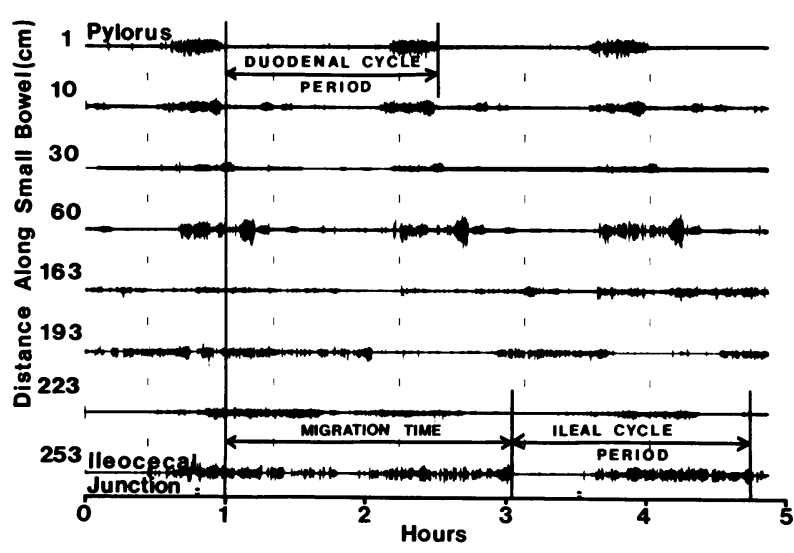

Figure 1 Fasting intestinal electrical activity recorded from eight electrodes in one dog. Three MMC, the duodenal and ileal cycle periods, and the MMC migration time are illustrated. each sample divided by the time period taken to collect the sample. Each sample consisted of the second dead space volume in any period plus the first dead space volume of the next period. This meant that the first dead space volume of the first sample was discarded and that sampling was continued after the final sample period to collect the second dead space volume of the last sample. The accuracy of the collections as a representation of the bile produced by the liver in a particular period is related to the error of the estimated dead space by: sampling error = estimated dead space error $/ 2$. Using this technique hepatic bile flow and bile acid secretion rates were determined at brief intervals. The duration of the interval was dependent on flow rate and ranged from 5 to $25 \mathrm{~min}$.

Total bile acid concentration in bile was measured using a modification of the enzymatic method of Talalay (39). The conversion of diphosphopyridine nucleotide (DPN) to its reduced form (DPNH) was measured in a spectrophotometer (Beckman model 35, Beckman Instruments, Inc., Fullerton, CA) at $340 \mathrm{~nm}$.

Radiolabeled $\left[{ }^{3} \mathrm{H}(\mathrm{G})\right]$ sodium taurocholate $(97 \%$ pure by TLC) and $\left[24-{ }^{14} \mathrm{C}\right]$ sodium taurocholate (99\% pure by TLC), both of which were used for bolus duodenal instillation, were obtained from New England Nuclear; Lachine, Quebec, Canada. $50 \mu \mathrm{l}$ of bile from each sample period was added to $15 \mathrm{ml}$ Multisol (Isolabs) and counted in a liquid scintillation counter (Beckman Instruments model LS9000). $\left[{ }^{3} \mathrm{H}\right]$ DPN and $\left[{ }^{14} \mathrm{C}\right]$ DPN were determined using an external standard and quench correction factor.

In each experiment, the bile flow in milliliters per minute, bile acid secretion rate in micromoles per minute and radiolabeled bile acid secretion rate in disintegrations per minute were calculated for each aliquot of bile collected. These values were then plotted in relationship to the motility recording with the vertical coordinate representing magnitude, and the horizontal coordinate representing the midpoint of the collection interval.

The mass of bile acid secreted per collection interval is the product of volume collected times bile acid concentration. In the bile acid pool washout experiments the mass of bile acid secreted per collection interval was expressed as a percentage the total bile acid pool collected over $6 \mathrm{~h}$. The cumulative percentage of the bile acid pool excreted during the migration time of the first MMC was calculated for each washout experiment.

\section{Analysis}

In each experiment the time at which peak bile flow, bile acid secretion, or radiolabeled bile acid secretion occurred was recorded as a percentage of the MMC migration time. The mean \pm 1 SD of the time of peak secretion was then calculated for each variable in each protocol. A Wilcoxon rank sum test was used to determine if the maximum and minimum values of bile flow or bile acid secretion during each migration time were significantly different within a protocol, or if the times of peak bile flow and bile acid secretion were significantly different within a protocol. The Kruskal Wallis test (the nonparametric equivalent of oneway analysis of variance) was used to determine if the time of peak bile flow, bile acid secretion, or radiolabeled bile acid were significantly different in the bile acid pool washout experiments.

To plot bile flow or bile acid secretion against time and in relation to the MMC, the migration time of each MMC cycle in which bile was sampled was used as a time base of $100 \%$ length. The midpoints of the collection intervals dur- 
ing which bile flow and bile acid secretion were measured were plotted as percentile locations on this standard time base. During each migration time the magnitude of each variable in each collection interval was plotted on the vertical axis as a normal variate [normal variate $=(x-$ mean of $x$ )/standard deviation of $x$ ]. The curve obtained was interpolated (using the method of linear interpolation) at 5\% intervals and the values obtained at each interpolation point for all the experiments in each protocol were combined as a mean \pm 1 SD to yield a composite curve. The results were graphed. This data transformation allowed precise comparison of fluctuating values during the migration of different MMC unbiased by the variable range of absolute levels encountered in different experiments.

In the three experimental protocols there were $39 \mathrm{MMC}$ during which bile was sampled. We used a previously validated technique (40) to define the distal propagation in time of the MMC within each protocol, and for all MMC combined. Briefly, for any MMC a logarithmic function relates location of end phase III activity to elapsed migration time. This relationship can be plotted with horizontal axis units of elapsed percent migration time, identical to the horizontal axis units against which bile flow and bile acid secretion rates were plotted. When plotted on the same graph it is possible to make precise temporal comparison of bile flow and bile acid secretion rates with the distal migration of MMC despite variability in the gut length and migration times.

Statistical analysis was done using the Statistical Analysis Systems Package of the University of Toronto Computing Services.

\section{RESULTS}

There was no significant difference $(P>0.05)$ between the three protocols with regard to the slopes of the regression lines defining the distal propagation of the MMC with time. Therefore, the data for all $39 \mathrm{MMC}$ was combined. Fig. 2 shows the line that defines the distal propagation of $\mathrm{MMC}$ as they travel from 0 to $100 \%$ of gut length in $100 \%$ of the migration time. The equation of the line is:

$$
\ln (T+1)=0.0466(D)+0.16,
$$

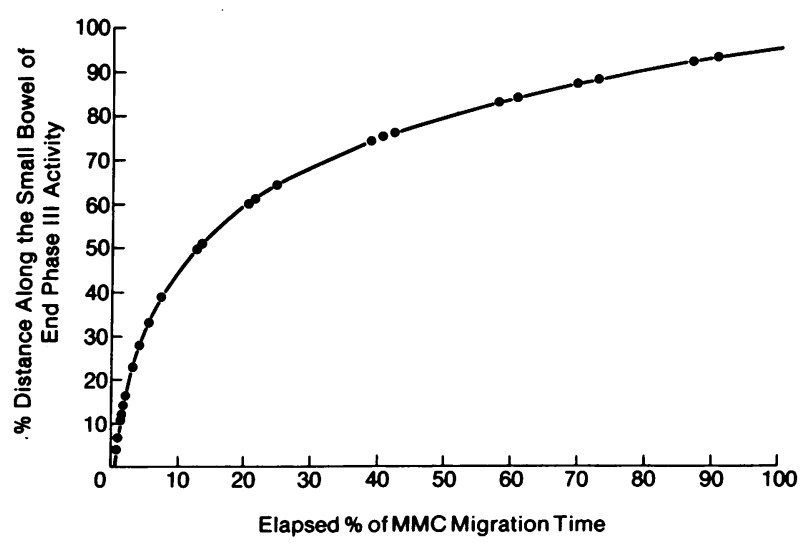

Figure 2 Regression line defining the distal propagation of the MMC. Percent distance migrated along the small bowel is plotted vs. elapsed percentage of the migration time. where ln is the natural logarithm, $\mathrm{T}$ is elapsed \% MMC migration time, $D$ is the \% distance end phase III of the MMC had traveled down the small bowel. The correlation coefficient of the relationship was 0.98 $(P<0.0001)$.

Intact enterohepatic circulation. Fig. 3 shows a 5$h$ record of fasting myoelectric activity in one dog. Electrode position along the gut is expressed on the vertical axis as a percentage of the distance along the small bowel, and time in hours is on the horizontal axis. The figure demonstrates the cyclic appearance of MMC in the duodenum and their sequential migration past more distal electrodes. During the recording, aliquots of bile were collected, sampled, and returned to the duodenum, thus leaving the enterohepatic circulation intact. Bile flow and bile acid secretion show repetitive fluctuations with peaks occurring in each case as phase III of the MMC passes the electrode located $83 \%$ of the distance along the small bowel, as highlighted by the arrowheads. There is one MMC whose distal migration stopped before reaching the electrode located $83 \%$ of the distance along the small bowel. Note that the peak bile acid secretion associated with that MMC is of lower amplitude.

In four experiments on two dogs in which the EHC was intact, bile flow, and bile acid secretion were monitored during the aboral migration of 14 MMC. During the distal migration of the MMC bile flow varied between a minimum of $0.17 \pm 0.04$ and a maximum of $0.38 \pm 0.07 \mathrm{ml} / \mathrm{min}(n=14)$. Bile acid secretion varied between a minimum of $15.1 \pm 3.9$ and a maximum of $40.4 \pm 10.7 \mu \mathrm{M} / \min (n=14)$. The minimum and $\max -$ imum values of either bile flow or bile acid secretion that occurred during each migration time were significantly different from each other $(P<0.01)$. The times at which peak bile flow and bile acid secretion occurred were $53.6 \pm 11.0$ and $49.8 \pm 11.8 \%(n=14)$, respectively of the migration time. The times of peak bile flow and bile acid secretion were not significantly different $(P>0.05)$. In Fig. 4 , the data on bile flow and bile acid secretion during the aboral migration of these $14 \mathrm{MMC}$ is combined and presented graphically. A separate line shows the propagation of MMC from 0 to $100 \%$ of gut length during $100 \%$ of the migration time. The maximum value of both bile flow and bile acid secretion occurs at $50-55 \%$ of elapsed migration time, the time at which the MMC had migrated $~ 80 \%$ of the distance along the small bowel. The increases of mean bile flow and mean bile acid secretion on either side of the central peak are due to the flow associated with MMC occurring before and after the index MMC.

Interrupted EHC with bile acid pool washout. Fig. 5 shows a recording of fasting small intestinal myoelectric activity during which the EHC was interrupted 


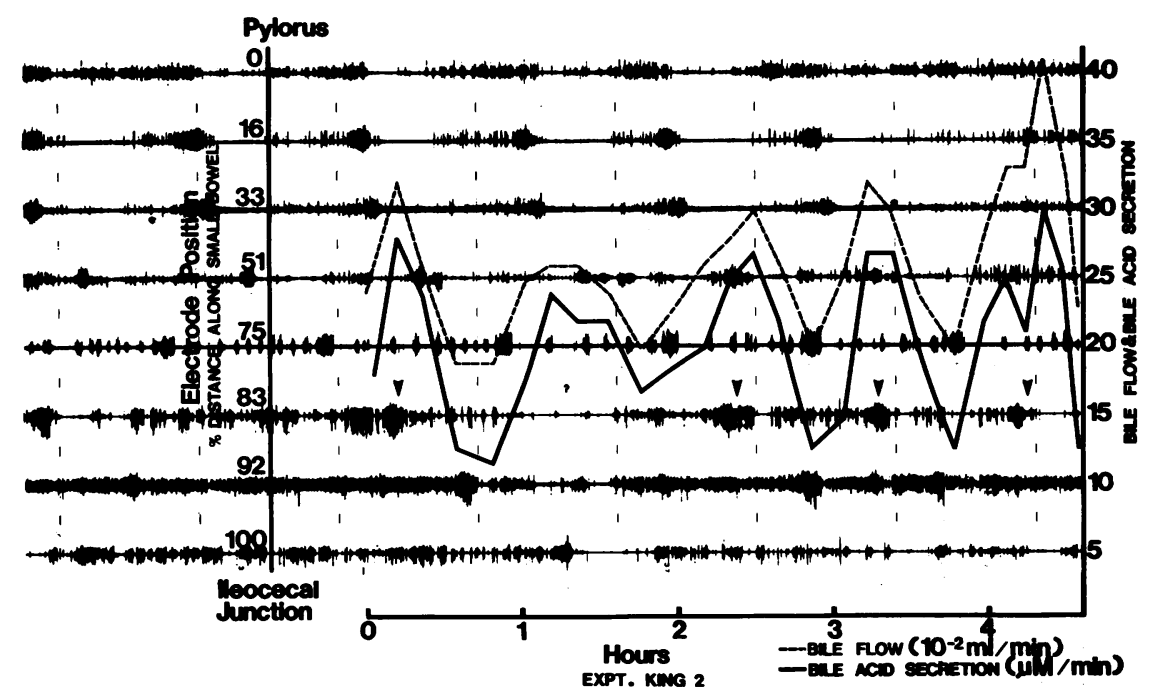

Figure 3 Bile flow and bile acid secretion with the enterohepatic circulation intact. Bile flow and bile acid secretion show repetitive fluctuations with peaks occurring (arrowheads) when the intense spiking activity of an MMC passes the electrode located $83 \%$ of the distance along the small bowel.

by collecting and retaining all hepatic biliary secretion for at least two complete MMC cycles and for a minimum of $6 \mathrm{~h}$. After bile collection commences, note that there is a peak of bile flow and bile acid secretion as phase III of the first MMC migrates from duodenum to terminal ileum; with the maximum secretion rates occurring as phase III of the MMC passed the electrode

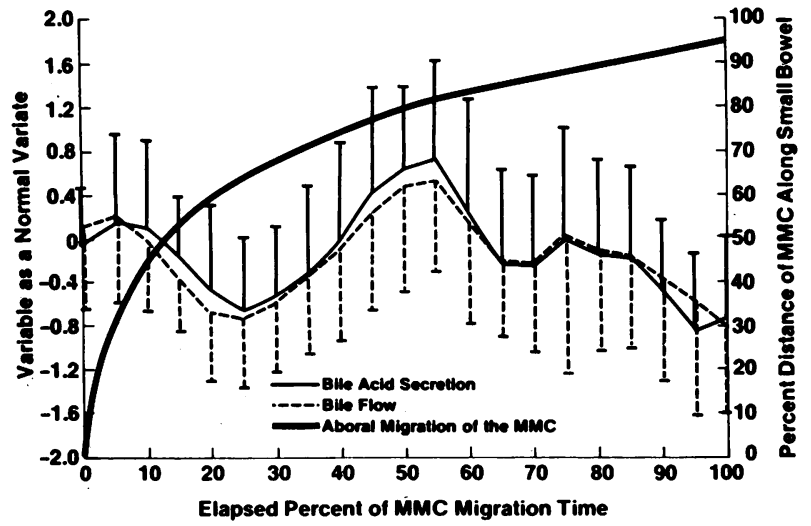

Figure 4 Bile flow and bile acid secretion during the aboral migration of $14 \mathrm{MMC}$ with the EHC intact. Bile flow and bile acid secretion (mean \pm 1 SD of the normal variates) are plotted against elapsed percent migration time. The regression line showing propagation of MMC from 0 to $100 \%$ of gut length during $100 \%$ of migration time is superimposed. Peak mean bile flow and bile acid secretion occur at 50-55\% of elapsed migration time, the time at which the MMC has migrated $\sim 80 \%$ of the distance along the small bowel. site $83 \%$ of the distance along bowel. This represents the bile secreted into the duodenum during the previous MMC and up to the time the EHC was interrupted. There was no subsequent peak of bile flow or bile acid secretion. After the intraluminal bile acid pool was delivered to the terminal ileum, by the first MMC, bile flow dropped to a steady state and bile acid secretion decreased to a minimum. The intraluminal bile acid pool was effectively cleared from the intestine after one passage of the MMC.

In Fig. 5 we further characterize the transport function of the MMC by instilling a bolus of radiolabeled sodium taurocholate into the duodenum at the same time bile collection began, just before phase III of the MMC passed through the duodenum. This was performed in 8 of the 12 bile acid pool washout experiments. In all cases, peak endogenous and exogenous radiolabeled bile acid secretion occurred together indicating that both were delivered to the terminal ileum simultaneously by the first MMC. In addition, both were cleared from the intestine by the first passage of an MMC.

In the 12 experiments in which the EHC was interrupted and the bile acid pool collected over $6 \mathrm{~h}$, bile flow varied between a minimum of $0.08 \pm 0.02$ and maximum of $0.32 \pm 0.08 \mathrm{ml} / \mathrm{min}(n=12)$. Bile acid secretion varied between a minimum of $0.9 \pm 0.3$ and a maximum of $39.4 \pm 13.5 \mu \mathrm{M} / \mathrm{min}(n=12)$. The minimum and maximum values of either bile flow or bile acid secretion that occurred during each 6 -h collection were significantly different from each other $(P<0.01)$. 


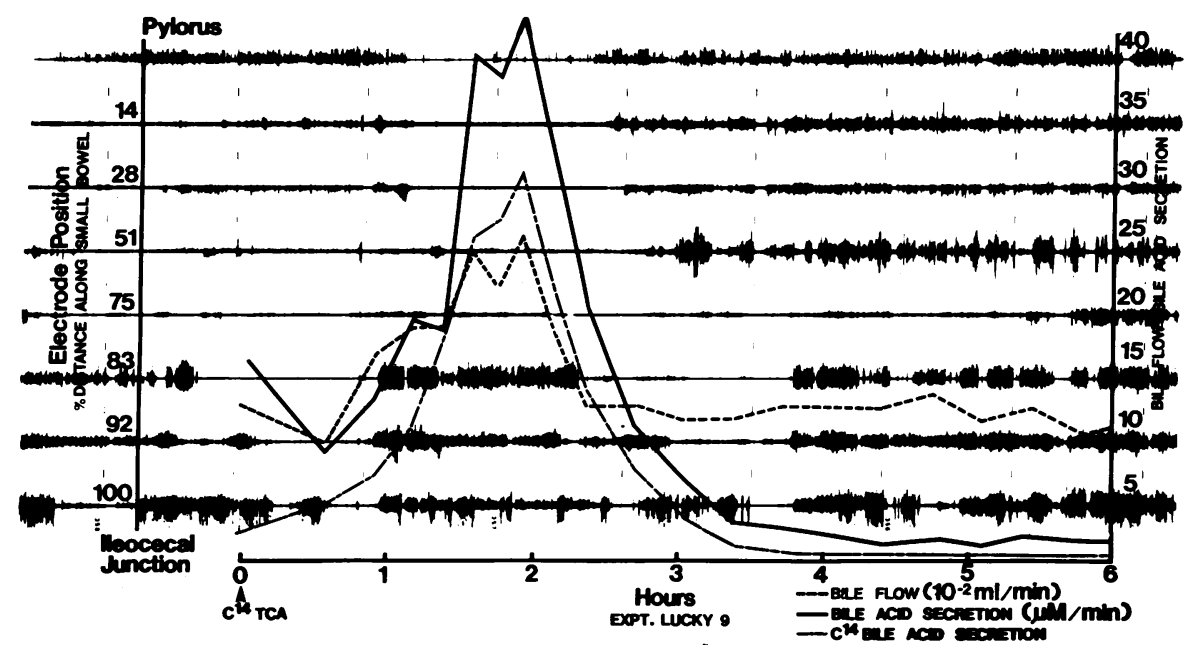

FIGURE 5 Bile flow and bile acid secretion after interruption of the EHC. A bolus of radiolabeled $\mathrm{Na}$ taurocholate was instilled into the duodenum at the same time bile collection began, just before the spiking activity of an MMC passed through the duodenum. Peak bile flow, endogenous bile acid, and radiolabeled bile acid secretion occur as phase III of that MMC pass the electrode site $83 \%$ of the distance along the bowel. Bile flow and bile acid secretion (endogenous and radiolabeled) then decrease to minimum constant levels.

The time at which peak bile flow and bile acid secretion occurred were $32.7 \pm 13.9$ and $33.1 \pm 16.5 \%$ ( $n$ $=12$ ), respectively, of the migration time. The times of peak bile flow and bile acid secretion occurred were not significantly different $(P=0.81)$. In the eight bile acid pool washout experiments in which radiolabeled sodium taurocholate was instilled into the duodenum prior to the passage of a duodenal MMC the time of peak isotope excretion was $29.6 \pm 18.4 \%$ of the migration time and was not significantly different from the time of peak bile flow or bile acid secretion $(P>0.05)$. The times of peak bile flow, bile acid secretion, and radiolabeled bile acid secretion corresponded to the time at which the MMC had migrated $70 \%$ of the distance along the small bowel. During the bile acid pool washout studies $88 \%$ of the bile acid pool collected was secreted during the passage of the first MMC, and this occurred within the first $2.4 \pm 0.4 \mathrm{~h}$.

The transport function of the MMC is further defined in Fig. 6. Here, the bolus of radiolabeled sodium taurocholate was instilled into the duodenum just after phase III of an MMC passed through the duodenum. Bile collection and interruption of the EHC began at the same time. As in the previous experiment, bile flow and endogenous bile acid secretion appear highly correlated and peak as phase III of the first MMC passes beyond the electrode located $75 \%$ of the distance along the small bowel. This first MMC again clears the endogenous bile acid pool in one pass. However, the bolus of radiolabeled sodium taurocholate was not secreted with the endogenous bile acid pool. It was secreted separately, and later, at a time when passage of the succeeding cycle of the MMC delivered it to the distal small intestine. In all seven experiments in which boluses of radiolabeled sodium taurocholate were instilled into the duodenum just after passage of the duodenal MMC the time of peak radiolabeled bile acid secretion occurred significantly later than the peak endogenous bile acid secretion $(P<0.01)$, and in relation to motor activity of the subsequent MMC. This indicates that the endogenous pool had been moved down the intestine with the first MMC; however, the radiolabeled bile acid instilled just after phase III of this MMC remained in the proximal small bowel and was not cleared to the ileal site of absorption until the subsequent MMC started in the duodenum and passed aborally down the intestine.

Interrupted EHC with continuous duodenal infusion of sodium taurocholate. Fig. 7 is another recording of fasting intestinal myoelectric activity. In this experiment, the enterohepatic circulation was interrupted, and during the study, the bile acid sodium taurocholate was infused into the duodenum at a constant rate of $20 \mu \mathrm{mol} / \mathrm{min}$. Despite the constant entry of bile acid into the duodenum biliary secretion again fluctuated. Peak bile flow and bile acid secretion occurred as phase III of each MMC passed beyond the electrode located $83 \%$ of the distance along the small bowel, as indicated by the arrowheads.

In four experiments on two dogs in which the EHC was interrupted during the continuous constant duodenal infusion of sodium taurocholate, bile flow, and bile acid secretion were monitored during the aboral migration of 13 MMC. During the distal migration of 


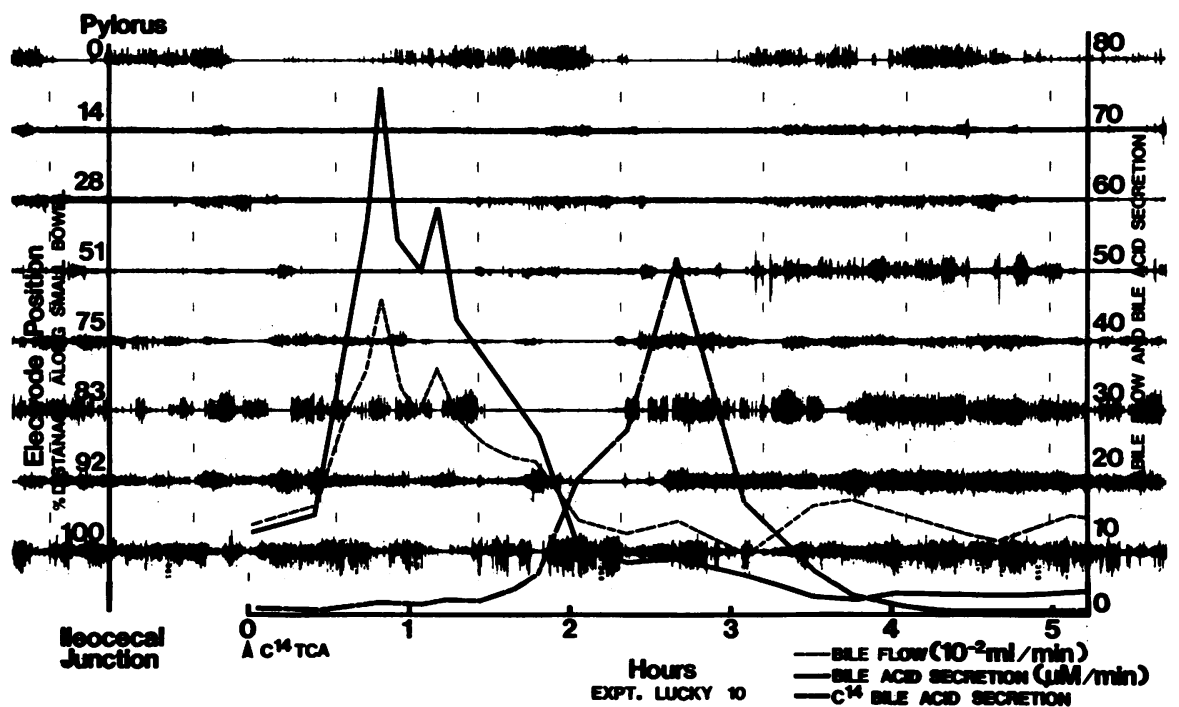

Figure 6 Bile flow, endogenous bile acid secretion, and radiolabeled $\mathrm{Na}$ taurocholate secretion in a 6-h bile acid pool washout experiment. A bolus of radiolabeled bile acid was instilled into the duodenum at the same time that bile collection began, just after the spiking activity of an MMC had passed through the duodenum. The endogenous bile acid pool was cleared by the first passing $\mathrm{MMC}$, however, the radiolabeled $\mathrm{Na}$ taurocholate was secreted in relation to motor activity of the subsequent MMC.

the MMC bile flow varied between a minimum of $0.12 \pm 0.04$ and a maximum of $0.29 \pm 0.08 \mathrm{ml} / \mathrm{min}(n$ $=13$ ). Bile acid secretion varied between a minimum of 7.0 \pm 2.7 and a maximum of $27 \pm 6.3 \mu \mathrm{M} / \mathrm{min}(n$
$=13$ ). The minimum and maximum values of either bile flow or bile acid secretion that occurred during each migration time were significantly different from each other $(P<0.01)$. The times at which peak bile

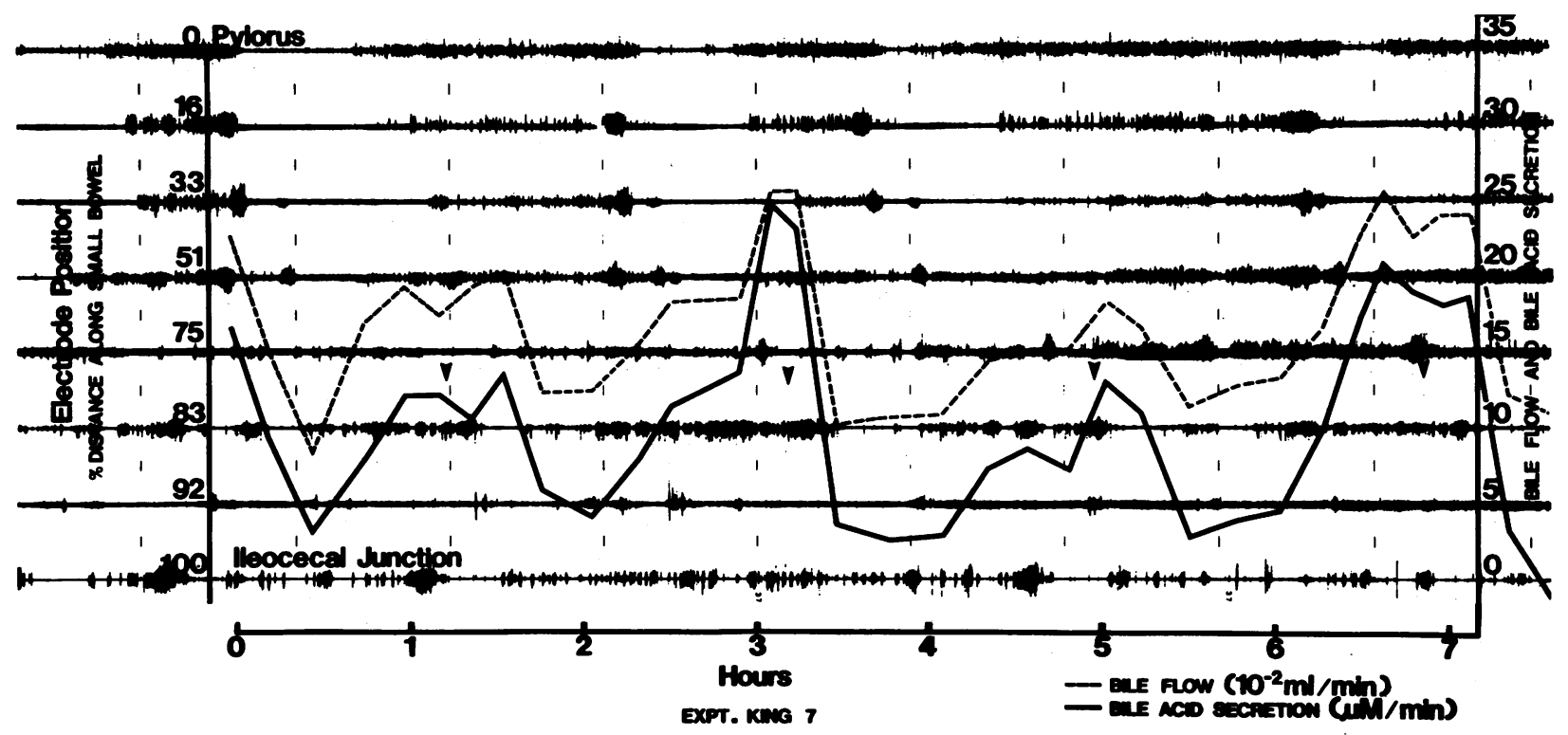

Figure 7 Bile flow and bile acid secretion with an interrupted EHC and continuous duodenal infusion of $\mathrm{Na}$ taurocholate. Despite the constant entry of bile salt into the duodenum, bile flow and bile acid secretion fluctuated, with peaks occurring (arrowheads) when the intense spiking activity of the MMC passed beyond the electrode located $83 \%$ of the distance along the small bowel. 
flow and bile acid secretion occurred were $50.9 \pm 9.9$ and $49.1 \pm 8.7 \%(n=13)$ of migration time, respectively. The times of peak bile flow and bile acid secretion were not significantly different $(P>0.05)$.

In Fig. 8 data from the $13 \mathrm{MMC}$ are presented graphically as before. Mean bile flow and mean bile acid secretion show similar fluctuation and peak at $45 \%$ of elapsed MMC migration time, the time at which the MMC had migrated $75-80 \%$ of the distance along the small bowel.

The mean time at which peak bile acid secretion occurred in the experiments with an intact EHC $(49.8 \pm 11.8 \%$ of migration time) or an interrupted EHC and continuous duodenal infusion of bile acids $(49.1 \pm 8.7 \%$ of migration time) were not significantly different $(P>0.05)$. However, the mean time at which peak bile acid secretion occurred in the bile acid pool washout experiments $(33.1 \pm 16.5 \%$ of migration time) was significantly different $(P<0.01)$ from the other two protocols. The peak of bile acid secretion occurred earlier in the bile acid pool washout experiments. This was also the case for the mean times of peak bile flow $(P<0.01)$.

\section{DISCUSSION}

These experiments have demonstrated that in the fasting state the transport of intestinal bile acids to the liver is rhythmic rather than continuous, and that the major determinant of these cyclical fluctuations is the

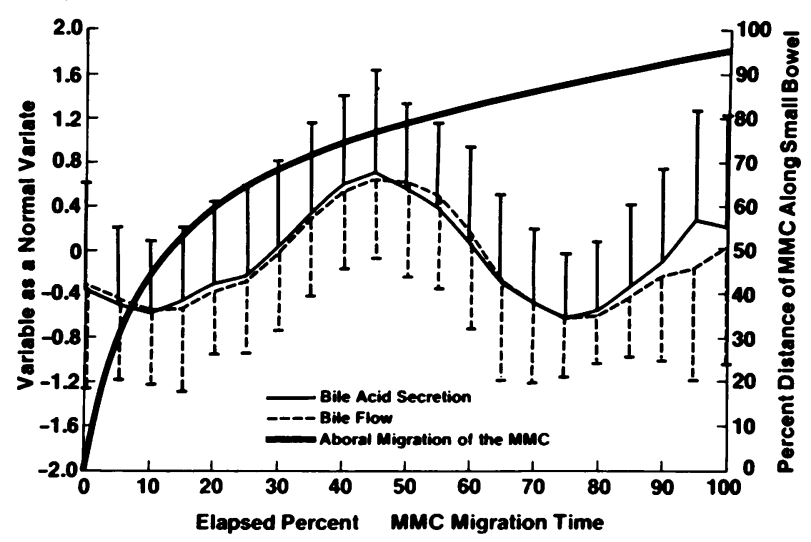

Figure 8 Bile flow and bile acid secretion during the aboral migration of $13 \mathrm{MMC}$ with the EHC interrupted and during the continuous constant duodenal infusion of sodium taurocholate. Bile flow and bile acid secretion (mean \pm 1 SD of the normal variates) are plotted against elapsed \% of the migration time. The regression line showing propagation of MMC from 0 to $100 \%$ of gut length during $100 \%$ of migration time is superimposed. Peak mean bile flow and bile acid secretion occur at $45 \%$ of elapsed migration time, the time at which the MMC had migrated $70-80 \%$ of the distance along the small bowel. periodic transport function of the MMC. Bile acids delivered into the duodenum prior to duodenal phase III of the MMC are transported aborally to their distal site of absorption by the first passing MMC. Bile acids delivered into the duodenum after passage of a duodenal phase III await the motor activity of the succeeding duodenal MMC for distal transport and absorption. This is true for endogenous bile acids in an intact EHC, and for exogenous bile acids delivered into the duodenum by a constant or bolus infusion. In all three experimental protocols, maximum bile flow and hepatic bile acid secretion occurred when phase III of the MMC propelled the intraluminal bile acid pool into the distal small bowel. The remarkable transport efficiency of this aborally propagating band of contractions is demonstrated by the first pass clearance from the small bowel of $88 \%$ of a 6 -h washout bile acid pool. Interruption of the EHC and removal of the bile acid pool resulted in nonfluctuating basal rates of bile flow and bile acid secretion.

It is well accepted that the circulation and localization of the bile acid pool within the EHC is determined by two active metabolic transport systems, two smooth muscle pumps, and the sphincter of Oddi (1). The metabolic transport systems in the hepatocyte and the terminal ileum are responsible for the obligate, efficient, and rapid, transfer of bile acids from the portal blood to bile canaliculus, and from the intestinal lumen to portal blood stream, respectively. The gallbladder is the only portion of the EHC with a significant ability to sequester and store bile acids, and during fasting part of the hepatic bile secreted is diverted into the gallbladder (41), while approximately one-half of the bile acid pool continues an enterohepatic circulation (10-13). The coordinated activity of the sphincter of Oddi, and the gallbladder pump regulates delivery of bile acids to the duodenum $(41,42)$. The motor pump of the small intestine controls delivery of intestinal bile acids to their site of absorption in the terminal ileum. In our experiments we removed the gallbladder pump and sphincter of Oddi from the system and dealt with the remaining small intestinal pump and the two metabolic transport systems. Transfer of bile acids through the two metabolic transport systems is extremely rapid. Bile acids instilled into the terminal ileum of experimental animals cause an increase in hepatic bile flow within minutes, and the $t_{1 / 2}$ for plasma disappearance of tracer doses of major conjugated and unconjugated bile acids injected intravenously in man is $<3 \mathrm{~min}(43)$. These times are small and constant compared to the longer and variable times for migration of the MMC from duodenum to the distal site of bile acid absorption. Thus, the metabolic transport systems do not themselves create fasting fluctuations in bile acid secretion, and in our animal model any variation in bile flow and bile acid secretion must be attributed to the 
fasting small intestinal pump, i.e. the migrating motor complex.

During fasting there are cyclical fluctuations in the rate of delivery of bile acids to the duodenum. In the intact fasting dog and human the peak delivery of bile acids into the duodenum is consistently associated with late phase II, or initial phase III of the duodenal MMC (23-26). Our results demonstrate that hepatic secretion and bile flow increased consistently with delivery of intestinal bile acids to the site of absorption in the distal small bowel. There was, however, no consistent relation of the increases in hepatic secretion to the duodenal phases of the MMC. Therefore, the delivery of bile acids to the liver and the subsequent rate of hepatic secretion is a function of the intestinal pump; but in the intact animal, delivery of bile acids into the duodenum must be dictated by the gallbladder and sphincter of Oddi, which also coordinate the time of delivery with the duodenal phases of the MMC.

Gallbladder contractions have been proposed as a possible cause of the fluctuations in fasting delivery of bile acids to the duodenum (24). Using a canine animal model, Itoh et al. demonstrated that cyclic tonic contraction of the gallbladder with a force $\sim 80 \%$ of postprandial contractions occur briefly during early duodenal phase II of the MMC (27). However, Peeters et al. have demonstrated fasting fluctuation of bile acid delivery into the duodenum in cholecystectomized patients (25). Thus gallbladder contractions may contribute to the fasting fluctuation of bile acid delivery to the duodenum, but they are not essential for such fluctuations. The sphincter of Oddi function is a major factor regulating gallbladder filling and emptying (44, 45 ), and several authors have proposed that the sphincter of Oddi also plays a major role in determining the fasting pattern of delivery of bile acids into the duodenum $(24,25)$. Ashkin et al. demonstrated that during fasting the sphincter of Oddi exerts a retarding influence on hepatic bile secretion and delivery of bile into the duodenum (44); however, no one has yet explored the relationship between sphincter of Oddi motility, the rate of delivery of bile acids to the duodenum, and the MMC.

As reported by Keane et al. the peak duodenal delivery of bile acids is quite discrete and has a duration of only $\sim 12 \mathrm{~min}(24)$. In our experiments, the fluctuations in hepatic biliary secretion were not as discrete as those associated with delivery into the duodenum, but took place over the entire migration time, an interval that was always $>100 \mathrm{~min}$. There are a number of reasons for the gradual, more prolonged fluctuation of hepatic secretion. Firstly, the fasting gut is not quiescent between aboral passages of phase III of the MMC. Prior to each phase III, there is a long interval of irregular contractions (phase II) that propel intraluminal contents, although not as effectively as phase III (22). This motor activity would gradually transport bile acids delivered into the duodenum towards the distal site of absorption, and thus splay out the peak secretion. Secondly, the site of active bile acid absorption in the terminal ileum is of considerable length (46). Absorption would begin as the MMC transported intraluminal bile acids into that segment, and would continue as the MMC completed its migration through the segment. Thirdly, bile acids may be passively absorbed from any segment of the intestinal tract (47). This is particularly true for the glycine-conjugated bile acids and those with higher pKa values $(46,47)$. For example, after total ileectomy, there is complete interruption of the EHC with respect to sodium taurocholate (48), a bile acid which at intestinal pH remains in an ionized form and therefore requires the active transport system of the terminal ileum for absorption. However, if the normally constituted bile acid pool is studied, even a two-thirds resection of distal small bowel is still associated with significant absorption of bile acids (35).

In the bile acid pool washout experiments, the time of peak of bile acid secretion occurred significantly earlier than in the other two protocols. The difference in results is not due to any alteration of the aboral migration with time of the MMC since there was no significant difference $(P<0.05)$ between the three protocols with regard to the distal propagation of the MMC with time. There was also no consistent difference in the mass of bile acids secreted during the migration time of MMC in the three protocols that might explain the results. The bile washout experiments were done in three dogs, two of which were different than the two dogs used for the intact and interrupted EHC experiments. It is possible that the site for active absorption of bile acids in the terminal ileum varied in the different groups of animals.

In the bile acid pool washout experiments the mean duodenal cycle period prior to interruption of the EHC was significantly shorter than the mean succeeding duodenal cycle period (118.7 $\pm 34.8 \mathrm{~min}$. vs. $170.9 \pm 71.0$ $\min , \boldsymbol{P}<0.05$ ), and the increased duration of the succeeding duodenal cycle period appeared to be due to a prolongation of phase II activity. During these experiments the common bile duct was cannulated and bile flow was returned to the duodenum during one control migration of an MMC. Then the EHC was interrupted by collecting and retaining all bile for a 6$h$ period. Thus the prolongation of the duodenal cycle period was associated with the interrupted delivery of bile acids to the duodenum. The possibility that the cyclic delivery of bile into the duodenum is a factor modulating fasting intestinal motor activity needs to be explored.

Fasting activity of the EHC is much more dynamic than previously suspected. The results of our paper 
have demonstrated the role of the MMC (the fasting intestinal pump) in producing fasting fluctuation in hepatic bile acid secretion. The normal individual spends a significant portion of each 24-h day in the fasted state, and during this time the MMC determines the enterohepatic cycling frequency of the nonsequestered bile acid pool. The return to the liver of bile acids reabsorbed from the intestine has a regulatory role in bile acid synthesis, through feedback inhibition on the rate of transformation of cholesterol to bile acids $(35,49-54)$. Therefore, the periodicity of the MMC may be an important factor modulating the 24-h bile acid secretion rate, bile acid pool size, and bile acid synthesis rate. The effect of the periodicity of the MMC upon these parameters has implications with regard to the pathophysiology of cholesterol cholelithiasis. Further experiments are required to document the specific effects of fasting motility of the gallbladder and sphincter of Oddi upon the EHC and its function.

\section{ACKNOWLEDGMENTS}

The authors are grateful for the assistance of $\mathrm{K}$. E. Hall, R. G. Ilson, H. Mui, C. Petrunka, and D. Valdez.

This research was supported by the Medical Research Council of Canada grants MA3353, MA4491, the Toronto Western Hospital Research Foundation, and the Elsie Watt Fund.

\section{REFERENCES}

1. Hofmann, A. F. 1977. The enterohepatic circulation of bile acids in man. Clin. Gastroenterol. 6: 3-24.

2. Abaurre, R., S. G. Gordon, J. G. Mann, and F. Kern Jr. 1969. The effect of ileal resection upon fasting bile salt pool size and composition. Gastroenterology. 57: 679688.

3. Shaffer, E. A., J. W. Braasch, and D. M. Small. 1972 Bile composition at and after surgery in normal persons and patients with gallstones. Influence of cholecystectomy. N. Engl. J. Med. 287: 1317-1322.

4. LaRusso, N. F., M. G. Korman, N. E. Hoffman, and A. F. Hofmann. 1974. Dynamics of the enterohepatic circulation of bile acids. Postprandial serum concentrations of conjugates of cholic acid in health, cholecystectomized patients, and patients with bile acid malabsorption. N. Engl. J. Med. 291: 689-692.

5. Soloway, R. D., and L. J. Schoenfield. 1975. Effects of meals and interruption of enterohepatic circulation on flow, lipid composition, and cholesterol saturation of bile in man after cholecystectomy. Am. J. Dig. Dis. 20: 99109.

6. Brunner, H., T. C. Northfield, A. F. Hofmann, V. L. W. Go, and W. H. J. Summerskill. 1974. Gastric emptying and secretion of bile acids, cholesterol, and pancreatic enzymes during digestion. Duodenal perfusion studies in healthy subjects. Mayo Clinic Proc. 49: 851-860.

7. Northfield, T. C., and A. F. Hofmann. 1975. Biliary lipids during three meals and an overnight fast. I. Relationship to bile acid pool size and cholesterol saturation of bile in gallstone and control subjects. Gut. 16: 1-11.

8. Ivy, A. C., and E. Oldberg. 1928. A hormone mechanism for gallbladder contraction and evacuation. Am. J. Physiol. 86: 599-613.
9. Vantrappen, G., J. Janssens, J. Hellemans, and Y. Ghoos. 1977. The interdigestive motor complex of normal subjects and patients with bacterial overgrowth. J. Clin. Invest. 59: 1158-1166.

10. O'Brien, J. J., E. A. Shaffer, L. F. Williams, D. M. Small, J. Lynn, and J. Wittenburg. 1974. A physiological model to study gallbladder function in primates. Gastroenterology. 67: 119-125.

11. Shaffer, E. A., M. Beaudoin, D. M. Small, J. O'Brien, and L. Williams. 1974. Relationship between gallbladder function and the enterohepatic circulation of bile salts during fasting. Gastroenterology. 66: 775.

12. van Berge Henegouwen, G. P., and A. F. Hofmann. 1978. Nocturnal gallbladder storage and emptying in gallstone patients and healthy subjects. Gastroenterology. 75: 879-885.

13. Mok, H. Y. J., K. von Bergmann, and S. M. Grundy 1980. Kinetics of the enterohepatic circulation during fasting: Biliary lipid secretion and gallbladder storage. Gastroenterology. 78: 1023-1033.

14. Szurszewski, J. H. 1969. A migrating electric complex of the canine small intestine. Am. J. Physiol. 217: 17571763.

15. Grivel, M. L., and Y. Ruckebusch. 1972. The propagation of segmental contractions along the small intestine. J. Physiol. 227: 611-625.

16. Code, C. F., and J. A. Marlett. 1975. The interdigestive myoelectric complex of the stomach and small bowel of dogs. J. Physiol. 216: 289-309.

17. Itoh, Z., R. Honda, I. Aizawa, S. Tukeuchi, K. Hiwatashi, and E. F. Couch. 1978. Interdigestive motor activity of the lower esophageal sphincter in the conscious dog. Dig. Dis. 23: 239-247.

18. Bueno, L., J. Fioramonti, and Y. Ruckebusch. 1977. Mechanisms of propulsion in the small intestine. Ann. Rech. Vet. 8: 293-301.

19. Bueno, L., J. Fioramonti, and Y. Ruckebusch. 1975. Rate of flow of digesta and electrical activity of the small intestine in dogs and sheep. J. Physiol. 249: 69-85.

20. Ruckebusch, Y., and J. Fioramonti, 1975. Electrical spiking activity and propulsion in small intestine in fed and fasted rats. Gastroenterology. 68: 1500-1508.

21. Summers, R. W., J. Helm, and J. Christensen. 1976. Intestinal propulsion in the dog. Gastroenterology. 70: 753-758.

22. Kerlin, P., A. Zinsmeister, and S. Philips. 1982. Relationship of motility to flow of contents in the human small intestine. Gastroenterology. 82: 701-706.

23. DiMagno, E. P., J. C. Hendricks, V. L. W. Go, and R. R. Dozois. 1979. Relationships among canine fasting pancreatic and biliary secretion, pancreatic duct pressure, and duodenal type III motor activity-Boldyreff revisited. Dig. Dis. Sci. 24: 689-693.

24. Keane, F. B., E. P. DiMagno, R. R. Dozois, and V. L. W. Go. 1980 . Relationships among canine interdigestive exocrine pancreatic and biliary flow, duodenal motor activity, plasma pancreatic polypeptide, and motilin. Gastroenterology. 78: 310-316.

25. Peeters, T. L., G. Vantrappen, and J. Janssens. 1980. Bile acid output and the interdigestive migrating motor complex in normals and in cholecystectomy patients. Gastroenterology. 79: 678-681.

26. Vantrappen, G. R., T. L. Peeters, and J. Janssens. 1979. The secretory component of the interdigestive migrating motor complex in man. Scand. J. Gastroenterol. 14: 663667.

27. Itoh, Z., and I. Takahashi. 1981. Periodic contraction of 
the canine gallbladder during the interdigestive state. Am. J. Physiol. 240: G183-G189.

28. Weisbrodt, N. W. 1974. Electrical and contractile activities of the small intestine of the cat. Am. J. Dig. Dis. 19: 93-99.

29. Ruckebusch, Y., L. Bueno. 1976. The effect of feeding on the motility of the stomach and small intestine in the pig. Br. J. Nutr. 35: 397-405.

30. Ruckebusch, Y. 1977. Elektromyographische analyse der dunndarmmotorik der haustieren. Zentralbl. Veterinaermed. Reihe. A. 24: 1-12.

31. Fleckenstein, P. 1978. Migrating electrical spike activity in the fasting human small intestine. Dig. Dis. 23: 769775 .

32. Scott, R. B., H. Y. Mui, T. Y. El-Sharkawy, and N. E. Diamant. 1981. Effect of the migrating myoelectric complex on the enterohepatic circulation of bile acids. Clin. Invest. Med. 4: 19B.

33. Scott, R. B., S. M. Strasberg, T. Y. El-Sharkawy, and N. E. Diamant. 1981. Effect of the migrating myoelectric complex (MMC) on the enterohepatic circulation (EHC) of bile acids. Hepatology. 2: 545.

34. Markowitz, J. 1964. Textbook of Experimental Surgery. Fifth edition. Williams and Wilkins, Baltimore.

35. Dowling, R. H., E. Mack, and D. M. Small. 1970. Effects of controlled interruption of the enterohepatic circulation of bile salts by biliary diversion and by ileal resection on bile salt secretion, synthesis, and pool size in the Rhesus monkey. J. Clin. Invest. 49: 232-242.

36. Haaki, W., and G. Paumgartner. 1973. Determination of the biliary dead space using 14-Taurocholate as a marker. Experientia (Basel). 29: 1091-1093.

37. Barnhart, J. L., and B. Combes. 1979. Apparent volume of the biliary tree in the dog. Can. J. Physiol. Pharmacol. 57: 524-528.

38. Strasberg, S. M., K. A. Siminovitch, R. G. Ilson. 1974 Bile Production in Fasted and Fed Primates. Ann. Surg. 180: 356-363.

39. Talalay, P. 1960. Enzymatic analysis of steroid hormones. Methods Biochem. Anal. 8: 119-143.

40. Scott, R. B., T. Y. El-Sharkawy, and N. E. Diamant 1982. Propagation of the canine migrating myoelectric complex-A mathematical model. Am. J. Physiol. In press

41. Scott, G. W. 1980. Biliary tract: Anatomy and pathophysiology. Scientific Foundations of Gastroenterology.
W. Sircus and A. N. Smith, editors. Wm. Heinemann Medical Books Ltd., London, England. 547-564.

42. Ryan, J. P. 1981. Motility of the gallbladder and biliary tree. In Physiology of the Gastrointestinal Tract. L. R. Johnson, editor. Raven Press, New York. 473-494.

43. Cowen, A. E., M. G. Korman, A. F. Hofmann, and P. J. Thomas. 1975. Plasma disappearance of radioactivity after intravenous injection of labelled bile acids in man. Gastroenterology. 68: 1567-1573.

44. Ashkin, J. R., D. T. Lyon, S. D. Shull, C. I. Wagner, and R. D. Soloway. 1978. Factors affecting delivery of bile into the duodenum in man. Gastroenterology. 74: 560565.

45. Scott, G. W., R. E. Smallwood, and S. Rowlands. 1975. Flow through the bile duct after cholecystectomy. Surg. Gyn. Obstet. 140: 912-918.

46. Lack, L., and I. M. Weiner. 1963. Intestinal absorption of bile salts and some biological implications. Fed. Proc. 22: 1334-1338

47. Dietschy, J. M., H. S. Salomon, and M. D. Siperstein. 1966. Bile acid metabolism. I. Studies on the mechanisms of intestinal transport. J. Clin. Invest. 45: 832-846.

48. Playoust, M. R., L. Lack, and I. M. Weiner. 1965. Effect of intestinal resection on bile salt absorption in dogs. Am. J. Physiol. 208: 363-369.

49. Eriksson, S. 1957. Biliary excretion of bile acids and cholesterol in bile fistula rats. Bile acids and steroids. In Proceedings of the Society for Experimental Biology and Medicine. 94: 578

50. Bergstrom, S., and H. Danielsson. 1958. On the regulation of bile acid formation in the rat liver. Acta Physiol. Scand. 43: 1-7.

51. Shefer, S., S. Hauser, I. Bekersky, and E. H. Mosbach 1969. Feedback regulation of bile acid biosynthesis in the rat. J. Lipid Res. 10: 646-655.

52. Mok, H. Y. I., K. von Bergmann, and S. M. Grundy. 1977. Regulation of pool size of bile acids in man. Gas troenterology. 73: 684-690.

53. Duane, W. C., and K. C. Hanson. 1978. Role of gallbladder emptying and small bowel transit in regulation of bile acid pool size in man. J. Lab. Clin. Med. 92: 858872.

54. Motson, R. W., K. J. Hammerman, W. H. Admirand, and L. W. Way. 1981. Effect of chronic changes in rate of enterohepatic cycling on bile acid kinetics and biliary lipid composition in the Rhesus monkey. Gastroenterology. 80: $655-660$ 